\title{
ON A TRIPLY GRADED KHOVANOV HOMOLOGY
}

\author{
KRZYSZTOF K. PUTYRA
}

\begin{abstract}
Cobordisms are naturally bigraded and we show that this grading extends to Khovanov homology, making it a triply graded theory. Although the new grading does not make the homology a stronger invariant, it can be used to show that odd Khovanov homology is multiplicative with respect to disjoint unions and connected sums of links; same results hold for the generalized Khovanov homology defined by the author in his previous work. We also examine the module structure on both odd and even Khovanov homology, in particular computing the effect of sliding a basepoint through a crossing on the integral homology.
\end{abstract}

\section{INTRODUCTION}

The category $\mathbf{2 C o b}$ of 2-dimensional cobordisms is usually considered as $\mathbb{Z}$-graded, with the degree function given by the Euler characteristic of a cobordism. It was shown in [Put08, Put13] that this degree can be split into two numbers, one counting merges and births, whereas the other splits and deaths:

$$
\begin{array}{ll}
\operatorname{deg}(\Omega)=(-1,0) & \operatorname{deg}(\theta)=(1,0) \\
\operatorname{deg}(\Omega)=(0,-1) & \operatorname{deg}(\ominus)=(0,1)
\end{array}
$$

Indeed, the only relations that affect the set of critical points either create or remove a pair birth-merge or split-death, which does not change the two numbers. Because of that one can try to enhance the construction of Khovanov homology $\mathcal{H}^{i, j}(L)$ [Kho99] to a triply graded homology $\widetilde{\mathcal{H}}(L)^{i, p, q}$ with

$$
\mathcal{H}^{i, j}(L)=\bigoplus_{p+q=j} \widetilde{\mathcal{H}}^{i, p, q}(L)
$$

Indeed, the chain complex computing $\mathcal{H}(L)$ is constructed from the cube of resolutions of a diagram $D$ of $L$, vertices and edges of which are labeled with collections of circles and cobordisms between them respectively. Shifting degrees of the vertices appropriately results in a cube of graded maps, from which one can obtain a chain complex of bigraded groups.

Unfortunately, one does not obtain a stronger invariant in this way, as after a normalization $\widetilde{\mathcal{H}}(L)^{i, p, q}=0$ unless $p=q$. This is the reason why the author dropped this idea in his earlier works [Put08, Put13] on unification of the Khovanov homology with its odd variant [ORS13].

Date: November 11, 2018.

Key words and phrases. Khovanov homology, odd Khovanov homology, disjoint union, connected sum, module structure. 
The additional grading, however, appeared to be a key ingredient to understand the odd and generalized Khovanov homologies of composite links. It is well known that the original Khovanov homology, which we call in this paper the even Khovanov homology, is multiplicative with both disjoint unions and connected sums of links [Kho99, Kho04], i.e.

$$
\mathcal{H}_{e v}\left(L \sqcup L^{\prime}\right) \cong \mathcal{H}_{e v}(L) \underset{\mathbb{Z}}{\hat{\otimes}} \mathcal{H}_{e v}\left(L^{\prime}\right) \quad \text { and } \quad \mathcal{H}_{e v}\left(L \# L^{\prime}\right) \cong \mathcal{H}_{e v}(L) \underset{A}{\hat{\otimes}} \mathcal{H}_{e v}\left(L^{\prime}\right),
$$

where $\hat{\otimes}$ denotes a derived tensor product and $A$ is the Khovanov's algebra associated to a circle. To construct such isomorphisms for the odd and generalized Khovanov homology one has to understand the role of sign assignments better - the naive tensor product of complexes for links $L$ and $L^{\prime}$ does not give a priori a chain complex for $L \sqcup L^{\prime}$. The new grading is the additional information that helps to deal with this situation. Namely, it tells us that the naive isomorphism $\mathcal{H}_{\text {odd }}\left(L \sqcup L^{\prime}\right) \longrightarrow \mathcal{H}_{\text {odd }}(L) \hat{\otimes} \mathcal{H}_{\text {odd }}\left(L^{\prime}\right)$, i.e. the one given by the identities on chain groups, is not the correct one (it is even not a chain map). Instead, it has to be twisted by certain signs, which are controlled by the new grading.

The above is enough to derive a formula for disjoint unions of links, but to compute homology of a connected sum a module structure on the homology must be specified. It is defined naturally at the level of link diagrams with basepoints: the action of the algebra $A$ is induced by merging a circle with the diagram at the basepoint. Although it works nicely in the even setting, there are several issues in the case of generalized Khovanov homology. For instance, the algebra associated to a circle is not associative. This can happen, as the product is not a graded map - once the degree is shifted accordingly one obtains an associative algebra $A^{\prime}$, tensor products over which are well-defined.

Sliding a basepoint on a link does not change the module structure on the even Khovanov homology up to an isomorphism, and we proof the same for the generalized variant. It is a bit surprising that the module structure behaves nicer for the odd Khovanov homology - sliding a basepoint though a crossing does not change the module structure at all. On the other hand, one cannot move a dot from one link component to another. Therefore, we follow the idea of [HN12] and we construct $c$ actions of $A^{\prime}$ on the homology of a $c$-component link. When computing homology of $L \# L^{\prime}$ one should choose the actions determined by the link components that are joined together.

Our construction of a triply graded Khovanov homology is not the only one. There is another construction expected to result in a triply graded chain complex coming from a filtration on the HOMFLYPT homology [Ab14], but so far it is not proven to be invariant under all Reidemeister moves. We do not know how it compares with our grading.

Outline. We begin with a brief description of the category of chronological cobordisms $\mathbb{k} \mathbf{C h C o b}$ and graded tensor categories, introduced in [Put13], which provide a framework for the construction of the generalized Khovanov homology $\mathcal{H}(L)$. The construction of the generalized Khovanov complex $K h(D)$ is presented in Section 3 following [Put13]. The only 
difference is in regarding it as an object graded by $\mathbb{Z} \times \mathbb{Z}$, instead of graded by integers as in the original construction. The section ends with a proof that all the homotopy equivalences used in [Put13] to prove invariance of $K h(D)$ under Reidemeister moves preserve the new grading. Then in Section 4 we show that the new grading does not lead to new invariants.

The main part of the paper begins in Section 5, in which we derive the formulas for $K h\left(L \sqcup L^{\prime}\right)$ and $K h\left(L \# L^{\prime}\right)$, first at the level of complexes in $\mathbb{k} \mathbf{C h C o b}$. The application of chronological TQFT functors and the formulas for homology is delayed till the end of Section 6, after we construct the module structure on homology. We compute here the result of sliding a basepoint through a crossing - this was done in [HN12] over $\mathbb{Z}_{2}$, but our map is defined over integers.

\section{Chronological TQFTs}

In this paper $\mathbb{k}$ will always stand for the ring $\mathbb{Z}\left[X, Y, Z^{ \pm 1}\right] /\left(X^{2}=Y^{2}=1\right)$.

Definition 2.1 (cf. [Put13]). Let $W$ be a cobordism with a Riemann metric. A chronology on $W$ consists of a Morse function $h: W \longrightarrow I$ that separates critical points, and a choice of an orientation of $E^{-}(p)$, the space of unstable directions in the gradient flow induced by $h$, at each critical point $p$. We require $h^{-1}(0)$ and $h^{-1}(1)$ to be the input and output of $W$ respectively.

A standard argument from Morse theory implies that every 2-dimensional chronological cobordism can be built from six surfaces:
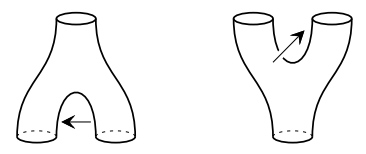

a merge a split a birth

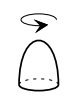

a positive a negative

death death

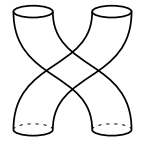

a twist

The little arrows visualize orientations of critical points. One merge and one split is sufficient, as the little arrow can be reversed by composing the cobordism with a twist.

Chronological cobordisms admit two disjoint unions: the 'left-then-right' $W$ 七列 and the 'right-then-left' one $W \triangleleft \Downarrow W^{\prime}$. Both are diffeomorphic to the standard disjoint union $W \sqcup W^{\prime}$, but to avoid a situation with two critical points at the same level one has to pull all critical points of $W$ below $\frac{1}{2}$ and those of $W^{\prime}$ over $\frac{1}{2}$ (for $\left.\downarrow \uparrow\right)$ or the other way (for $\left.\downarrow \downarrow\right)$ :

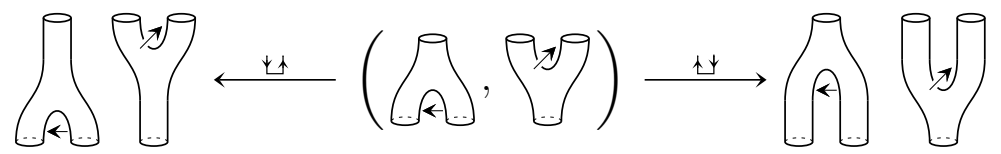

Likewise, there are two versions of a connected sum $W \# W^{\prime}$ and $W \# W^{\prime}$ :

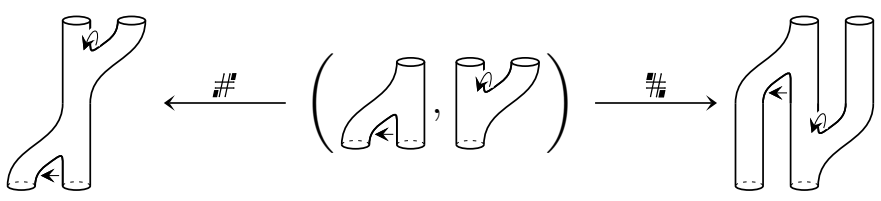


However, this operation requires more choices - a vertical line at each cobordism, along which they are glued together. We shall make this choice implicitly.

Definition 2.2. Define the degree $\operatorname{deg} W \in \mathbb{Z} \times \mathbb{Z}$ of a chronological cobordism $W$ by setting

$$
\operatorname{deg} W:=(\# \text { births }- \text { \#merges, \#deaths }- \text { \#splits }) \text {. }
$$

The chronological degree is clearly additive with respect to composition of chronological cobordisms as well as both disjoint unions and connected sums.

Lemma 2.3. Let $W$ be a chronological cobordism of degree $\operatorname{deg} W=(a, b)$ with $n$ inputs and $m$ outputs. Then $a+n=b+m$.

Proof. Straightforward, by checking for generating cobordisms (1).

Let $\mathbb{k} \mathbf{C h C o b}$ be a $\mathbb{k}$-linear category with finite disjoint unions of circles as objects, and formal $\mathbb{k}$-linear combinations of 2-dimensional chronological cobordisms as morphisms, modulo the following chronological relations:

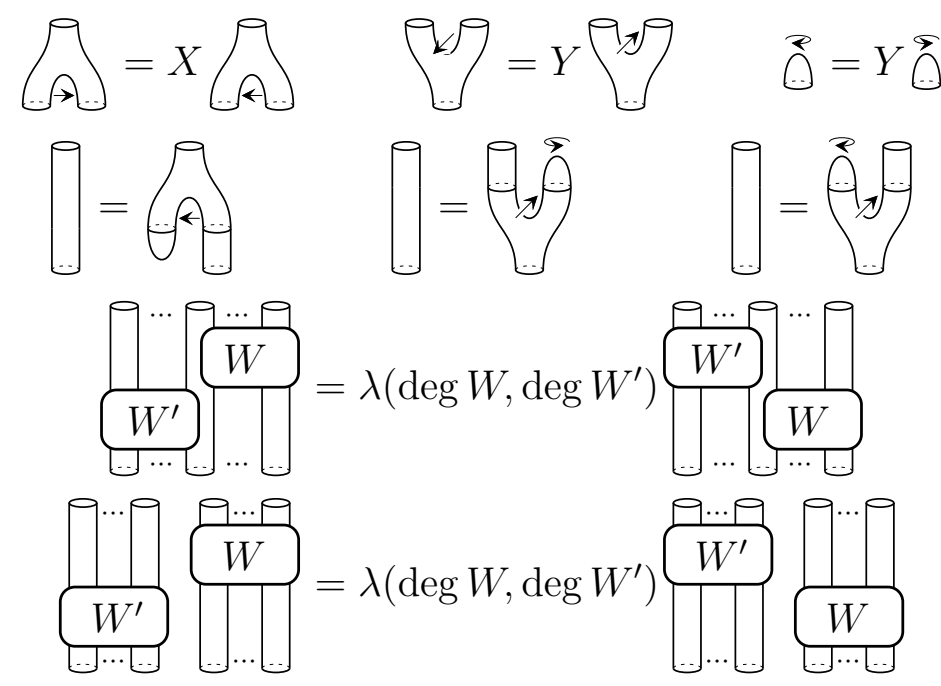

where $W$ and $W^{\prime}$ stand for any cobordisms, and $\lambda\left(a, b, a^{\prime}, b^{\prime}\right):=X^{a a^{\prime}} Y^{b b^{\prime}} Z^{a b^{\prime}-a^{\prime} b}$. Notice that the following associativity and Frobenius-type relations are special cases of (5):
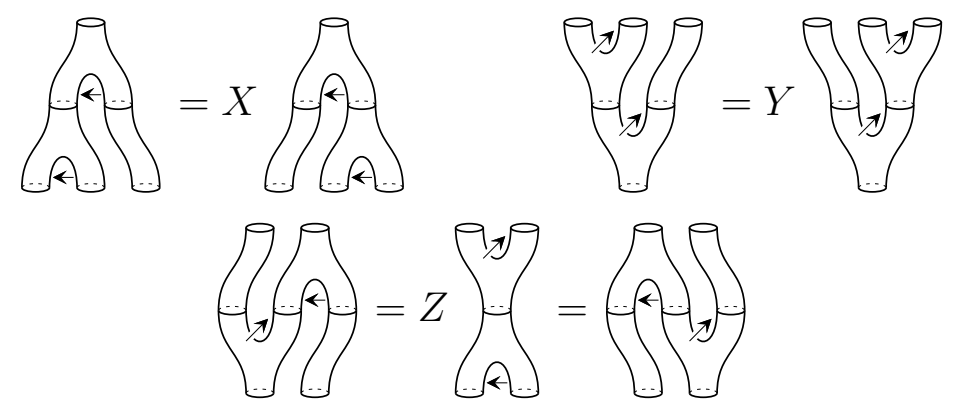

We proved in [Put13] the following non-degeneracy result for $\mathbb{k} \mathbf{C h C o b}$. 
Theorem 2.4. Suppose $k W=0$ for a chronological cobordism $W$ and a nonzero $k \in \mathbb{k}$. Then $W$ has either positive genus or at least two closed components, and $k$ is divisible by $(X Y-1)$. In particular, cobordisms cannot be annihilated by monomials.

Consider now the category $\mathbf{M o d}_{\mathbb{k}}$ of $\mathbb{k}$-modules graded by the group $\mathbb{Z} \times \mathbb{Z}$. We redefine the tensor product by setting for homogeneous homomorphisms $f$ and $g$

$$
(f \otimes g)(m \otimes n):=\lambda(\operatorname{deg} g, \operatorname{deg} m) f(m) \otimes g(n),
$$

where $\lambda\left(a, b, a^{\prime}, b^{\prime}\right)=X^{a a^{\prime}} Y^{b b^{\prime}} Z^{a b^{\prime}-a^{\prime} b}$ is defined as for $\mathbb{k} \mathbf{C h C o b}$. One checks directly that

$$
\left(f^{\prime} \otimes g^{\prime}\right) \circ(f \otimes g)=\lambda\left(\operatorname{deg} g^{\prime}, \operatorname{deg} f\right)\left(f^{\prime} \circ f\right) \otimes\left(g^{\prime} \circ g\right) .
$$

Hence, $\mathbf{M o d}_{\mathbb{k}}$ is a graded tensor category in the sense of [Put13]. There is a symmetry $\tau_{M, N}: M \otimes N \longrightarrow N \otimes M$ given by the formula $\tau_{M, N}(m \otimes n)=\lambda(\operatorname{deg} m, \operatorname{deg} n) n \otimes m$ for homogeneous elements $m \in M$ and $n \in N$.

A linear category is said to be graded by an abelian group $G$ if its morphism spaces are $G$-graded modules, and the grading is preserved by composition of morphisms. We also require an additive family of degree shift functors $A \longmapsto A\{g\}$ parametrized by $g \in G$, i.e. $A\{g\}\{h\}=A\{g+h\}$, such that the modules $\operatorname{Mor}(A\{m\}, B\{n\})$ and $\operatorname{Mor}(A, B)$ are naturally isomorphic up to grading: if a morphism $f \in \operatorname{Mor}(A, B)$ has degree $d$, then $\operatorname{deg} f=d+n-m$ when regarded as an element of $\operatorname{Mor}(A\{m\}, B\{n\})$.

The category $\mathbf{M o d}_{\mathbb{k}}$ is clearly graded by $\mathbb{Z} \times \mathbb{Z}$, but $\mathbb{k} \mathbf{C h C o b}$ is not - it lacks the degree shift functors. We introduce them formally by replacing the objects of $\mathbb{k} \mathbf{C h C o b}$ with symbols $\Sigma\{a, b\}$, where $\Sigma$ is a finite disjoint union of circles and $a, b \in \mathbb{Z}$. The degree of a chronological cobordism is extended over the new morphisms in a natural way:

$$
\operatorname{deg}\left(\Sigma\{a, b\} \stackrel{W}{\longrightarrow} \Sigma^{\prime}\left\{a^{\prime}, b^{\prime}\right\}\right):=\operatorname{deg}\left(\Sigma \stackrel{W}{\longrightarrow} \Sigma^{\prime}\right)+\left(a^{\prime}-a, b^{\prime}-b\right) .
$$

For example, the following morphism has degree $(0,0)$ :

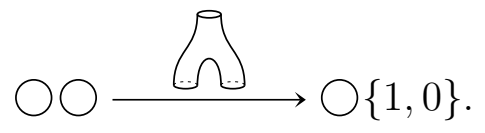

We reserve the symbol $\mathbb{k} \mathbf{C h C o b}$ for the subcategory of $\mathbb{k} \mathbf{C h C o b}$ spanned by morphisms of degree $(0,0)$. It is an abelian category.

Definition 2.5. A chronological TQFT is a graded functor $\mathcal{F}: \mathbb{k} \mathbf{C h C o b} \longrightarrow \mathbf{M o d}_{\mathbb{k}}$ that maps $₫$ into the graded tensor product $\otimes$ and the twist $\&$ into the symmetry $\tau$.

Example 2.6. We defined in [Put13] a chronological TQFT $\mathcal{F}: \mathbb{k} \mathbf{C h C o b} \longrightarrow$ Mod $_{\mathbb{k}}$, which maps a circle to a $\mathbb{k}$-module $A$ freely degenerated by $v_{+}$in degree $(1,0)$ and $v_{-}$in degree $(0,-1)$. On generating cobordisms $\mathcal{F}$ is defined as follows:

$$
\mathcal{F}(\Omega): A \otimes A \longrightarrow A, \quad \begin{cases}v_{+} \otimes v_{+} \longmapsto v_{+}, & v_{+} \otimes v_{-} \longmapsto v_{-}, \\ v_{-} \otimes v_{-} \longmapsto 0, & v_{-} \otimes v_{+} \longmapsto X Z v_{-}\end{cases}
$$




$$
\begin{aligned}
& \mathcal{F}\left(\left[\jmath^{\top} \rho\right): A \longrightarrow A \otimes A, \quad\left\{\begin{array}{l}
v_{+} \longmapsto v_{-} \otimes v_{+}+Y Z v_{+} \otimes v_{-}, \\
v_{-} \longmapsto v_{-} \otimes v_{-},
\end{array}\right.\right. \\
& \mathcal{F}(\theta): \mathbb{k} \longrightarrow A, \quad\left\{1 \longmapsto v_{+},\right. \\
& \mathcal{F}(\stackrel{\gtrless}{\odot}): A \longrightarrow \mathbb{k}, \quad\left\{\begin{array}{l}
v_{+} \longmapsto 0 \\
v_{-} \longmapsto 1
\end{array}\right.
\end{aligned}
$$

It is easy to see that $\mathcal{F}$ is a graded functor.

Remark 2.7. Given a $\mathbb{k}$-algebra $R$ we define likewise categories $R$ ChCob and Mod $_{R}$ together with a chronological TQFT $\mathcal{F}_{R}: R \mathbf{C h C o b} \longrightarrow \mathbf{M o d}_{R}$. In particular, if we consider $\mathbb{Z}$ as a trivial module, i.e. $X, Y$, and $Z$ act as the identity, $\mathbb{Z} \mathbf{C h C o b}$ is the linear extension of ordinary cobordisms - the relations $(3)-(6)$ become equalities - and $\mathcal{F}_{\mathbb{Z}}$ is the Khovanov's functor [Kho99].

Each of the parameters $X, Y$ and $Z$ is invertible, so that there are eight $\mathbb{k}$-algebra structures on the ring $\mathbb{Z}$. We shall distinguish two of them:

- $\mathbb{Z}_{e v}$, on which all $X, Y$, and $Z$ act trivially, and

- $\mathbb{Z}_{\text {odd }}$, on which $X$ and $Z$ act trivially, but $Y$ acts as -1 .

We call them the even and odd integers respectively. Both are quotients of $\mathbb{Z}_{\pi}:=\mathbb{Z}[\pi] /\left(\pi^{2}-1\right)$, on which $X$ and $Z$ act trivially, but $Y \cdot x:=\pi x$.

\section{The Generalized Khovanov COMPlex}

We shall now briefly describe the construction of the generalized Khovanov complex. We encourage the reader to refer to Fig. 1 frequently while reading this section; it illustrates the construction for the right-handed trefoil.

3.1. The cube of resolutions. Fix a link diagram $D$ and enumerate its crossings. Given a sequence $\xi=\left(\xi_{1}, \ldots, \xi_{n}\right)$, where $\xi_{i} \in\{0,1\}$ and $n$ is the number of crossings in $D$, let $D_{\xi}$ be the collection of circles obtained by resolving each crossing according to the following rule:

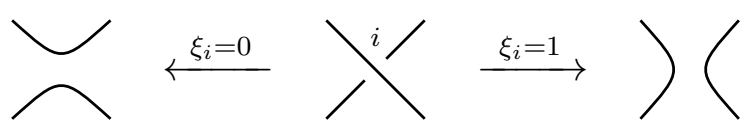

The diagrams $D_{\xi}$ decorate vertices of an $n$-dimensional cube $\mathcal{I}(D)$, called the cube of resolutions of $D$. Let $\|\xi\|:=\xi_{1}+\ldots+\xi_{n}$ be the weight of the vertex $\xi$. Consider an edge $\zeta: \xi \longrightarrow \xi^{\prime}$ oriented towards the vertex with higher weight. The diagrams $D_{\xi}$ and $D_{\xi^{\prime}}$ differ only in a smoothing of a single crossing, and we decorate the edge $\zeta$ with the simplest possible cobordisms between the two pictures $D_{\zeta} \subset \mathbb{R}^{2} \times I$, which is a vertical surface except a small 


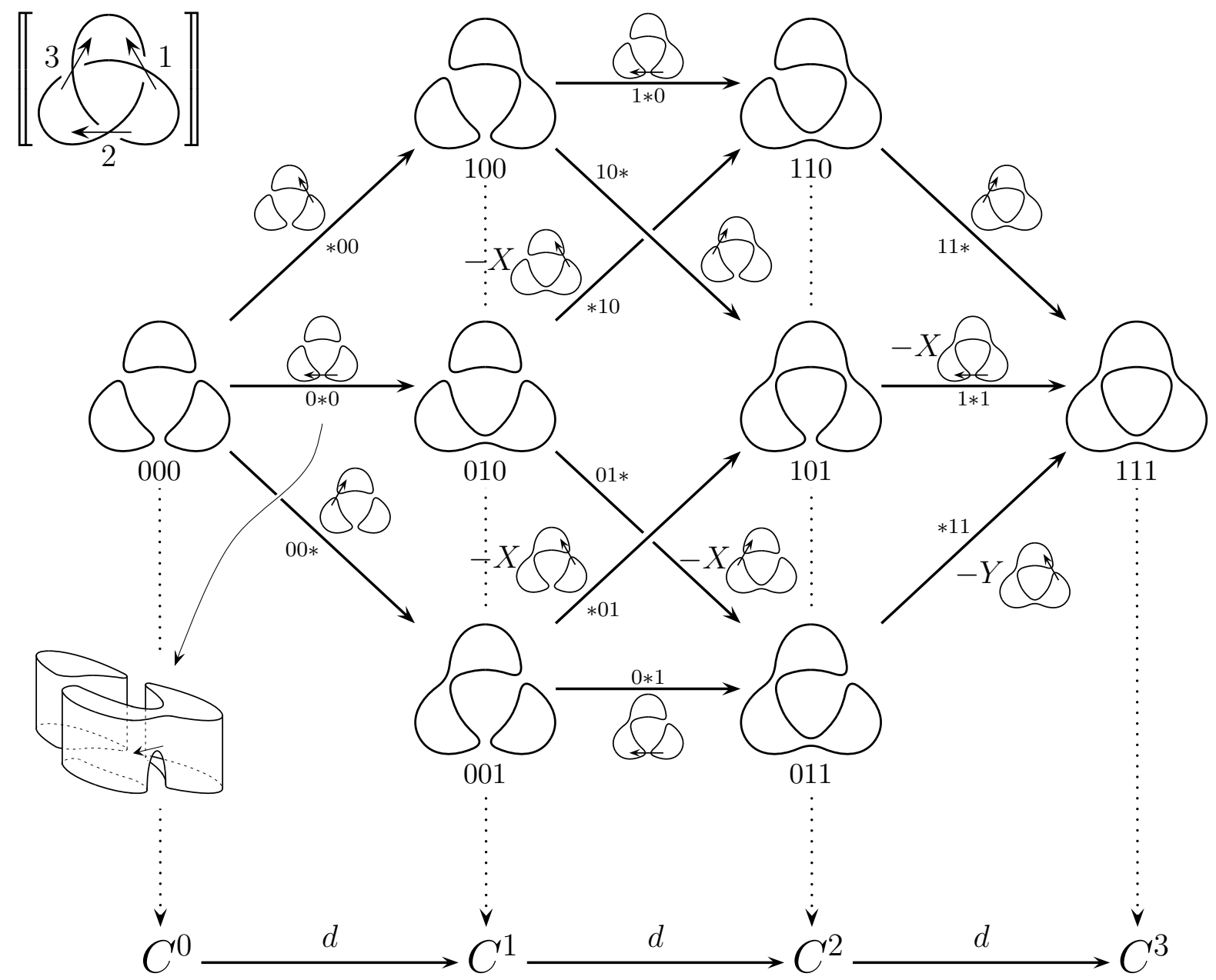

Figure 1. The cube of resolutions and the generalized Khovanov complex for the right-handed trefoil.

neighborhood of the crossing whose smoothing is changed-here we insert a saddle [. ${ }^{1}$ Decorate crossings of the link diagram $D$ with small arrows, which connects the two arcs in type 0 resolution - they determine uniquely orientations of saddle points of the cobordisms $D_{\zeta}$, so that $\mathcal{I}(D)$ can be regarded as a diagram in the category $\mathbb{k} \mathbf{C h C o b}$.

\footnotetext{
${ }^{1}$ In Fig. 1 we use the surgery description for cobordisms: the input circles together with an arc, a surgery along which results in the output circles. The arc is oriented, which determines an orientation of the saddle. A $3 \mathrm{D}$ picture of one cobordism is provided in the left bottom corner.
} 
3.2. Sign assignments. The cube $\mathcal{I}(D)$ does not commute, but there is a cubical cocycle $\psi \in C^{2}\left(I^{n} ; \mathbb{k}^{*}\right)$, the commutativity obstruction, such that for every face $S$ of the form

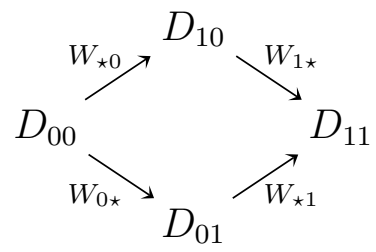

the twisted commutativity $W_{1 \star} W_{\star 0}=\psi(S) W_{\star 1} W_{0 \star}$ holds. Given a face $S$ as in (17) consider the resolution $D_{00}$ with two arrows orienting the four cobordisms. After removing isolated circles, i.e. those not touched by any arrow, we end up with one of the diagrams listed in Tab. 1; the table defines the value of $\psi(S)$. In the view of Theorem 2.4 in most cases $\psi(S)$ is determined by the relations (3), (5), and (6). The only exceptions are the one circle configurations at the bottom - in these cases the two compositions in (17) represent diffeomorphic cobordisms with positive genus, so that $\psi(S)$ can be either 1 or $X Y$.

If $\delta \epsilon=-\psi$ for a cubical cochain $\epsilon \in C^{1}\left(I^{n} ; \mathbb{k}^{*}\right)$, the corrected cube $\mathcal{I}^{\epsilon}(D)$, in which each cobordism $D_{\zeta}$ is multiplied by $\epsilon(\zeta)$, anticommutes. We call such a 1-cochain a sign assignment following [ORS13]. It is shown in [Put13] that such a sign assignment always exists and is unique up to an isomorphism of cubes.

3.3. Grading. Let $\ell\left(D_{\xi}\right)$ be the number of circles in the state $D_{\xi}$ of a link diagram $D$. We refine the cube of resolutions $\mathcal{I}^{\epsilon}(D)$ to the graded cube $\mathcal{I}_{g r}^{\epsilon}(D)$ by setting

$$
\mathcal{I}_{g r}^{\epsilon}(D)_{\xi}:=D_{\xi}\left\{\operatorname{deg} W_{\xi}\right\}=D_{\xi}\left\{\frac{\|\xi\|-\ell(\xi)+\ell_{0}}{2}, \frac{\|\xi\|+\ell(\xi)-\ell_{0}}{2}\right\}
$$

where $\|\xi\|:=\xi_{1}+\cdots+\xi_{n}$ is the weight of the vertex $\xi=\left(\xi_{1}, \ldots, \xi_{n}\right), W_{\xi}$ is a cobordism encoded by any directed path from the initial vertex $(0 \cdots 0)$ to $\xi$, and $\ell_{0}:=\ell\left(D_{0}\right)$ is the number of circles in the initial state (all crossing are smoothed in type 0 ). Commutativity of the cube guarantees $\operatorname{deg} W_{\xi}$ is independent of the path chosen, and it can be computed from the number of input and output circles using Lemma 2.3. All morphisms in $\mathcal{I}_{\text {gr }}^{\epsilon}(D)$ are graded. ${ }^{2}$

The integral grading from [Kho99, ORS13, Put13], wich we write as $\operatorname{deg}_{q}$, can be recovered from this construction by adding the two components of deg. For cobordisms it is equal to the Euler characteristic: $\operatorname{deg}_{q} W=\chi(W)$. We shall refer to it as the collapsed grading.

3.4. The complex. The generalized Khovanov complex is constructed in the additive closure $\operatorname{Mat}(\mathbb{k} \mathbf{C h C o b})$ of the category $\mathbb{k} \mathbf{C h C o b}$, which objects are finite sequence (vectors) of objects from $\mathbb{k} \mathbf{C h C o b}$, and morphisms are matrices with linear combinations of chronological cobordisms as its entries; a direct sum in $\left.\operatorname{Mat}(\mathbb{k} \mathbf{C h C o b})_{0}\right)$ is realized by concatenation of sequences.

\footnotetext{
${ }^{2}$ A morphism $f$ is graded if $\operatorname{deg} f=(0,0)$.
} 


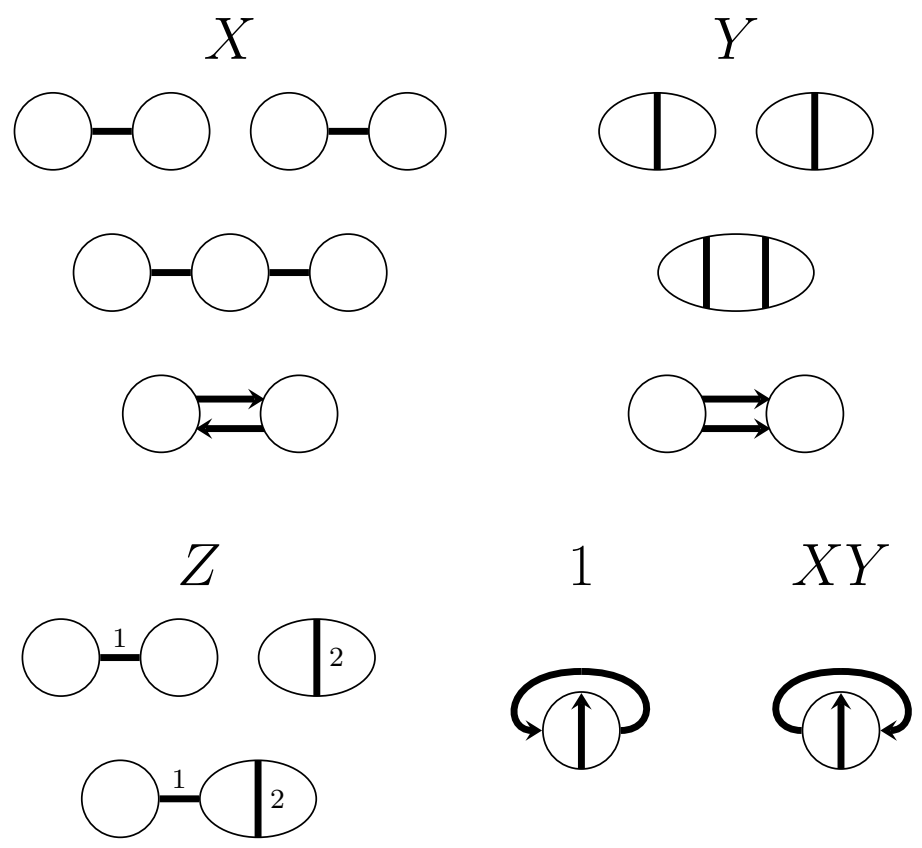

TABLE 1. Diagrams for faces that can appear in a cube of resolutions, grouped by values of the commutativity obstruction $\psi$. Thin lines are the input circles and thick arrows visualize saddle points. Orientations of the arrows are omitted if $\psi$ does not depend on them. The small numbers 1 and 2 in the two configurations placed under the letter $Z$ indicate an initial order of critical points (the upper path in (17)); take $Z^{-1}$ for the opposite one.

Definition 3.1. Let $D$ be a link diagram with enumerated and oriented crossings. Given a sign assignment $\epsilon$ for the cube $\mathcal{I}(D)$ we define the generalized Khovanov bracket as the chain complex $\llbracket D \rrbracket_{\epsilon}$ in the category $\left.\operatorname{Mat}(\mathbb{k} \mathbf{C h C o b})_{0}\right)$ with

$$
\llbracket D \rrbracket_{\epsilon}^{i}:=\bigoplus_{|\xi|=i} D_{\xi}\left\{\frac{\|\xi\|-\ell(\xi)+\ell_{0}}{2}, \frac{\|\xi\|+\ell(\xi)-\ell_{0}}{2}\right\},\left.\quad d^{i}\right|_{D_{\xi}}:=\sum_{\zeta: \xi \rightarrow \xi^{\prime}} \epsilon(\zeta) D_{\zeta} .
$$

The generalized Khovanov complex $K h(D)$ is obtained from $\llbracket D \rrbracket_{\epsilon}$ by shifting both homological and internal grading: $K h^{i}(D):=\llbracket D \rrbracket_{\epsilon}^{i+n_{-}}\left\{\frac{n_{+}-\ell_{0}}{2}-n_{-}, \frac{n_{+}+\ell_{0}}{2}-n_{-}\right\}$.

One can think of $\llbracket D \rrbracket_{\epsilon}$ as the cube $\mathcal{I}_{g r}^{\epsilon}(D)$ collapsed along diagonals, which is illustrated by dotted arrows in Fig. 1.

Remark 3.2. It seems more natural to shift the degrees in $\mathcal{I}(D)$ by $\left(\frac{\|\xi\|-\ell(\xi)}{2}, \frac{\|\xi\|+\ell(\xi)}{2}\right)$ without the terms $\ell_{0}$, as the latter is removed in $K h(D)$. However, the numbers $\|\xi\| \pm \ell(\xi)$ can be odd leading to a grading by half-integers, in which case the formula (6) requires square roots of $X, Y$, and $Z$ to make sense. We shall not encounter this problem with the chosen convention - all construction will be defined for the bracket, and a global degree shift does not introduce any additional signs. 
Theorem 3.3. The homotopy type of the generalized Khovanov complex $K h(D)$ is a link invariant, when regarded as a complex in the category Mat $\left(\mathbb{k} \mathbf{C h} \mathbf{C o b}_{0}\right)$ modulo the following three local relations:
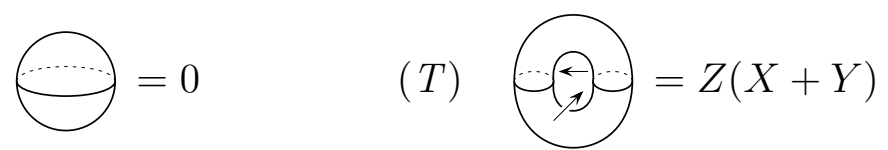

$(4 T u)$

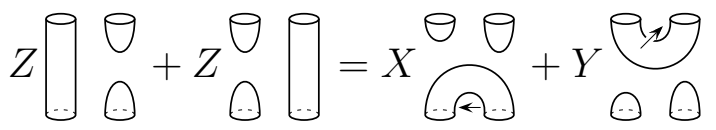

in which all deaths are oriented clockwise.

Proof. We showed in [Put13] that the isomorphism class of $\llbracket D \rrbracket_{\epsilon}$ depends only on the diagram $D$, and all isomorphisms involved are induced by cobordisms with no critical points. Hence, this still holds in the bigraded framework. The relations $S, T, 4 T u$ are clearly homogeneous, so that the quotient category is still graded. It remains to show the chain homotopy equivalences from [Put13] are graded.

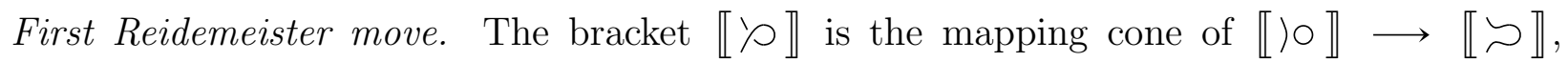
the chain map induced by the edges in $\mathcal{I}(`)$ associated with the distinguished crossing.

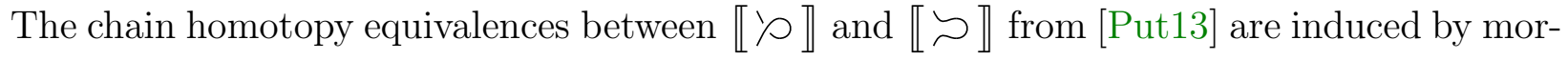
phisms of cubes $f: \mathcal{I}(\supset) \rightleftarrows \mathcal{I}() \circ): g$ with components visualized in the diagram below. Here, $\epsilon$ comes from the sign assignment used to build $\llbracket ` \rrbracket$, and $\alpha \in \mathbb{k}$ is chosen for each component of $f$ and $g$ separately to make them commute with other edge morphisms in the cubes. Because $g: \llbracket \curlyvee \rrbracket \longrightarrow \llbracket つ \rrbracket$ is induced by a death, $\operatorname{deg} g=(0,1)$. Likewise, the two components of $f: \llbracket つ \rrbracket \longrightarrow \llbracket \curvearrowright \rrbracket$ are cobordisms of degree $(0,-1)$. Since )o has

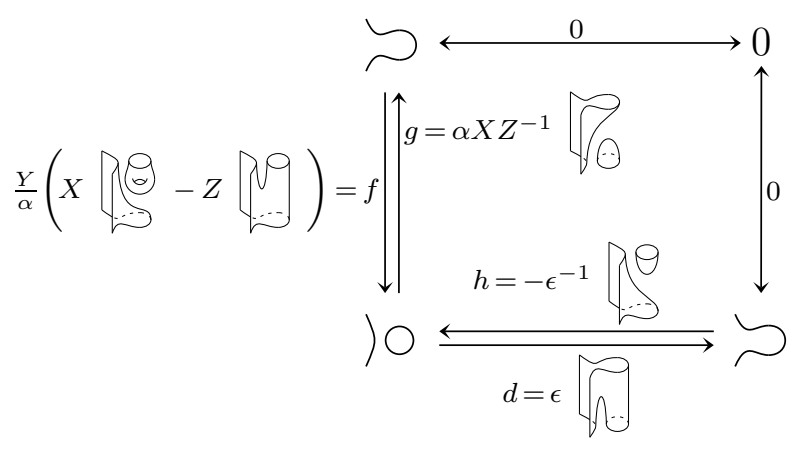
one more circle than $\checkmark$, both $f$ and $g$ become graded morphisms when the degree shifts are applied.

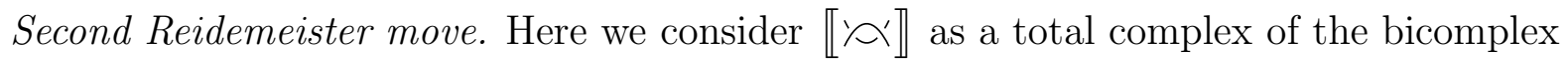

$$
0 \longrightarrow \llbracket) \approx \rrbracket \longrightarrow \llbracket) \circ(\rrbracket \oplus \llbracket \approx \rrbracket \longrightarrow \llbracket \curvearrowright(\rrbracket \longrightarrow 0 .
$$

The chain homotopy equivalences between $\llbracket \curlywedge^{\prime} \rrbracket$ and $\llbracket \asymp \rrbracket$ are then induced by maps shown in Fig. 2. The morphisms $\asymp \rightleftarrows \approx$ are graded: both diagrams have the same number of circles, and the difference between heights of corresponding vertices in cubes $(\approx$ has one more type 1 resolution than $\asymp)$ is compensated by the difference between numbers of crossings. The other components of $f$ and $g$ are also graded morphisms, which follows from their definitions

$$
(\asymp \stackrel{f}{\longrightarrow}) \circ():=\left(\asymp \stackrel{\mathrm{id}}{\longrightarrow} \approx \stackrel{d_{1 *}}{\longrightarrow} \precsim\left(\stackrel{h_{* 1}}{\longrightarrow}\right) \circ()\right.
$$




$$
\left.() \circ(\stackrel{g}{\longrightarrow} \asymp):=() \circ\left(\stackrel{h_{0 *}}{\longrightarrow}\right) \propto \stackrel{d_{* 0}}{\longrightarrow} \approx \stackrel{\mathrm{id}}{\longrightarrow} \asymp\right)
$$

and the fact that both $d$ and $h$ are graded morphisms in the graded complex ( $d$ is graded by the construction and $h$ has the opposite degree to $d$ ).
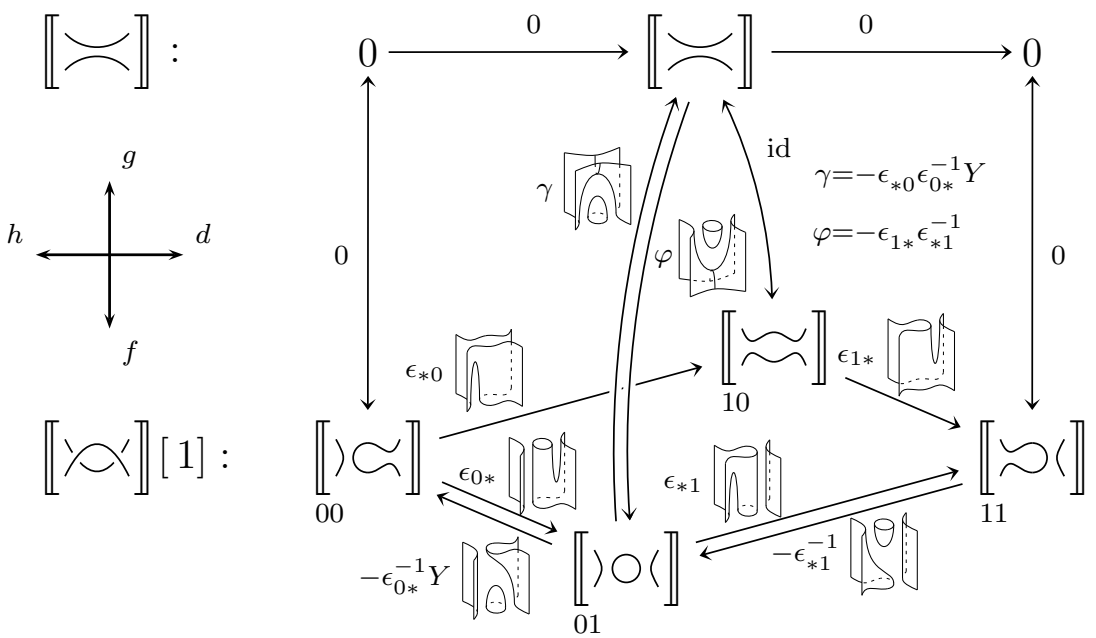

Figure 2. Chain homotopy equivalences for the second Reidemeister move.

Third Reidemeister move. This case follows from a strictly algebraic argument: the complex

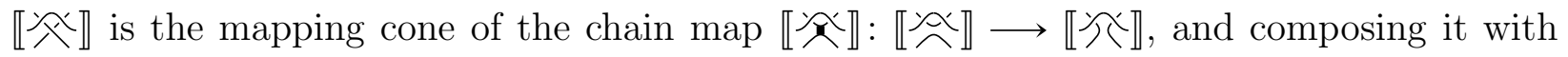
the chain homotopy equivalence $f: \llbracket \smile \rrbracket \longrightarrow \llbracket \stackrel{\sim}{\frown} \rrbracket$ does not change the homotopy type of

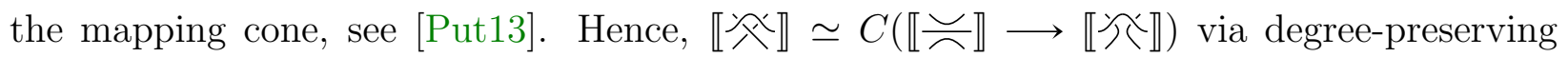
chain homotopy equivalences, and similarly for $\llbracket \underset{x}{ } \rrbracket$.

\section{Homology}

Applying a chronological TQFT $\mathcal{F}: \mathbb{k} \mathbf{C h C o b} \longrightarrow \operatorname{Mod}_{\mathrm{k}}$ to the complex $K h(D)$ results in a chain complex $\mathcal{F} K h(D)$ of bigraded $\mathbb{k}$-modules. If $\mathcal{F}$ preserves the relations $S, T$, and $4 T u$ from Theorem 3.3, the homology of the chain complex $\mathcal{F} K h(D)$ is invariant under Reidemeister moves. In particular, we can choose the TQFT from Example 2.6 and write $\mathcal{H}(L)$ for homology of the corresponding chain complex. As usual, given a $\mathbb{k}$-module $M$ we define $\mathcal{H}(L ; M)$ as the homology of the chain complex $\mathcal{F} K h(D) \otimes M$.

Proposition 4.1 (cf. [Put13]). Choose a link $L$ and consider $\mathcal{H}(L)$ with the collapsed integral grading. Then the following holds:

(1) the graded Euler characteristic $\chi(\mathcal{H}(L))$ is the Jones polynomial of $L$,

(2) $\mathcal{H}\left(L ; \mathbb{Z}_{e v}\right)$ is the Khovanov homology of $L$ [Kho99], and

(3) $\mathcal{H}\left(L ; \mathbb{Z}_{\text {odd }}\right)$ is the odd Khovanov homology of $L$ [ORS13]. 
One may expect that the new gradation on $K h(D)$ results in a stronger invariant. However, as long as $\mathcal{F}(\bigcirc)$ is generated in degrees $(1,0)$ and $(0,-1)$ the bigraded graded chain complex $\mathcal{F} K h(D)$ carries no more information than the one with the collapsed grading.

Lemma 4.2. Let $\mathcal{F}: \mathbb{k} \mathbf{C h C o b} \longrightarrow \operatorname{Mod}_{\mathbb{k}}$ be a chronological TQFT such that $A:=\mathcal{F}(\bigcirc)$ is supported in degrees $(1,0)$ and $(0,-1)$ only. Then $\mathcal{F} K h^{i}(D)_{p, q} \neq 0$ only if $p=q$.

Proof. Let $k:=\ell\left(D_{\xi}\right)$ be the number of circles in a state $D_{\xi}$ of the diagram $D$. A homogeneous element $u \in \mathcal{F}\left(D_{\xi}\right)=A^{\otimes k}$ has degree $\operatorname{deg} u=(d, d-k)$ for some $d \in \mathbb{Z}$. When regarded as an element of $\mathcal{F} K h(D)$ we have to adjust the degree by $\left(\frac{\|\xi\|-k+n_{-}}{2}-n_{-}, \frac{\|\xi\|+k+n_{+}}{2}-n_{-}\right)$. Hence, $u \in \mathcal{F} K h^{i}(D)_{p, p}$ with $p=\frac{\|\xi\|-k+n_{+}}{2}+d-n_{-}$.

Corollary 4.3. The graded Euler characteristic of $\mathcal{H}(L)$, regarded as a tripple graded $\mathbb{k}$ module, is the Jones polynomial of $L$ evaluated at $\sqrt{u v}$.

Despite this unfortunate result, the additional grading is actually useful, which will become clear in the next section.

\section{KhOVANOV COMPLEXES FOR COMPOSITE LINKS}

Given complexes $C$ and $C^{\prime}$ in a graded monoidal category, which differentials $d: C_{i} \longrightarrow C_{i+1}$ and $d^{\prime}: C_{i}^{\prime} \longrightarrow C_{i+1}^{\prime}$ are graded, we define their tensor product as the chain complex $C \otimes C^{\prime}$ satisfying

$$
\begin{aligned}
\left(C \otimes C^{\prime}\right)_{i} & :=\bigoplus_{p+q=i} C_{p} \otimes C_{q}^{\prime}, \\
\left.d\right|_{C_{p} \otimes C_{q}^{\prime}} & :=d \otimes \mathrm{id}+(-1)^{p} \mathrm{id} \otimes d^{\prime} .
\end{aligned}
$$

The role of the tensor product in $\mathbb{k} \mathbf{C h C o b}$ is played by the disjoint union $\downarrow \downarrow$, in which case $K h^{p}(D) \downarrow \Downarrow K h^{q}\left(D^{\prime}\right)$ is represented by diagrams $D_{\xi} \sqcup D_{\xi^{\prime}}^{\prime}$ with $\|\xi\|=p$ and $\left\|\xi^{\prime}\right\|=q$, which can be seen as resolutions of $D \sqcup D^{\prime}$. However, the morphisms $d \sqcup$ id and id $\sqcup d$ in $K h\left(D \sqcup D^{\prime}\right)$ do not commute. The reason they do in $K h(D) \downarrow \downarrow K h\left(D^{\prime}\right)$ is the way the degree shifts are applied: the objects $D_{\xi}\{a, b\} \sqcup D_{\xi^{\prime}}^{\prime}\left\{a^{\prime}, b^{\prime}\right\}$ and $\left(D_{\xi} \sqcup D_{\xi^{\prime}}^{\prime}\right)\left\{a+a^{\prime}, b+b^{\prime}\right\}$ are isomorphic but not equal.

Definition 5.1. Let $X$ be an object of a $G$-graded monoidal category and $g \in G$. The degree shift isomorphism $i_{g}: X \longrightarrow X\{g\}$ is the degree $g$ map corresponding to $\operatorname{id}_{X} \in \operatorname{Mor}(X, X)$ under the natural bijection $\operatorname{Mor}(X, X\{g\}) \cong \operatorname{Mor}(X, X)$.

We use the degree shift isomorphisms to identify both $X\{g\} \otimes Y\{h\}$ and $(X \otimes Y)\{g+h\}$ with $X \otimes Y$. There is some choice for the first isomorphism: we can take either $i_{g}^{-1} \otimes i_{h}^{-1}$ or $\left(i_{g} \otimes i_{h}\right)^{-1}$, and a short computation reveals the two morphisms are not equal:

$$
\left(i_{g}^{-1} \otimes i_{h}^{-1}\right) \circ\left(i_{g} \otimes i_{h}\right)=\lambda(-h, g)\left(i_{g}^{-1} \circ i_{g}\right) \otimes\left(i_{h}^{-1} \circ i_{h}\right)=\lambda(-h, g) \text { id } .
$$


The following result suggests to choose $i_{g}^{-1} \otimes i_{h}^{-1}$, as signs will then appear only when a nontrivial morphism occurs as the second factor (compare it with (10)).

Lemma 5.2. Choose an object $X$ and a morphism $f: A \longrightarrow B$ of degree $\operatorname{deg} f=a-b$ in a G-graded monoidal category. Then the following diagrams commute

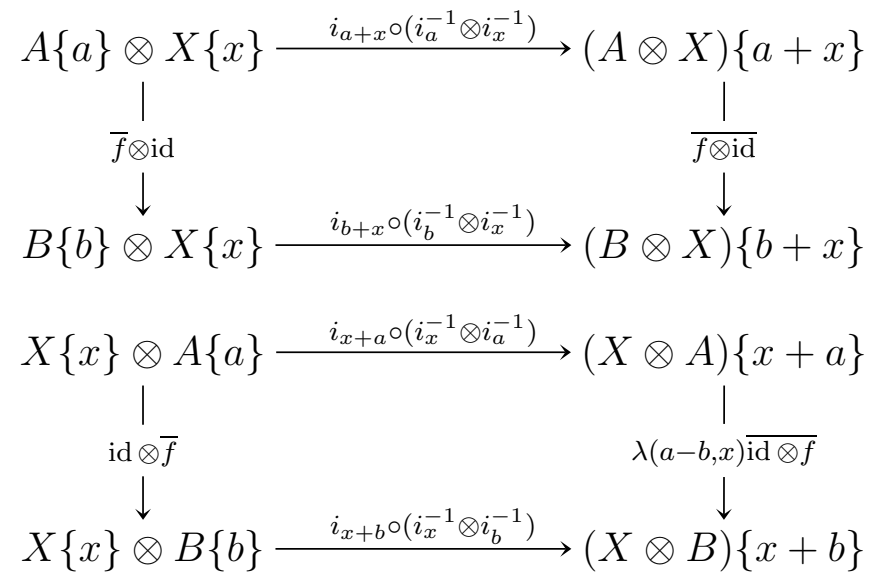

where $\vec{\cdot}$ denotes appropriate conjugations by degree shift isomorphisms $i_{\bullet}$.

Proof. Follows directly from (10).

Choose cubes $\mathcal{I}$ and $\mathcal{I}^{\prime}$ of dimensions $n$ and $n^{\prime}$ respectively. We define their product as a cube $\mathcal{I} \otimes \mathcal{I}^{\prime}$ of dimension $n+n^{\prime}$ with vertices decorated with tensor products

$$
\left(\mathcal{I} \otimes \mathcal{I}^{\prime}\right)\left(\xi \xi^{\prime}\right):=\mathcal{I}(\xi) \otimes \mathcal{I}^{\prime}\left(\xi^{\prime}\right)
$$

and edges labeled by the original maps tensored with identity morphisms:

$$
\begin{aligned}
\left(\mathcal{I} \otimes \mathcal{I}^{\prime}\right)\left(\zeta \xi^{\prime}\right) & :=\mathcal{I}(\zeta) \otimes \operatorname{id}_{\mathcal{I}^{\prime}\left(\xi^{\prime}\right)} \\
\left(\mathcal{I} \otimes \mathcal{I}^{\prime}\right)\left(\xi \zeta^{\prime}\right) & :=\operatorname{id}_{\mathcal{I}(\xi)} \otimes \mathcal{I}^{\prime}\left(\zeta^{\prime}\right)
\end{aligned}
$$

As usual, $\xi$ and $\xi^{\prime}$ stand for sequences encoding vertices, whereas $\zeta$ and $\zeta^{\prime}$ encode edges. Notice that $\mathcal{I} \otimes \mathcal{I}^{\prime}$ may not commute even if both $\mathcal{I}$ and $\mathcal{I}^{\prime}$ do.

Consider now link diagrams $D, D^{\prime}$ and their cubes of resolutions $\mathcal{I}_{g r}^{\epsilon}(D), \mathcal{I}_{g r}^{\epsilon^{\prime}}\left(D^{\prime}\right)$ corrected by certain sign assignments $\epsilon \in C^{1}\left(I^{n} ; \mathbb{k}^{*} *\right)$ and $\epsilon^{\prime} \in C^{1}\left(I^{n^{\prime}} ; \mathbb{k}^{*}\right)$, where $n$ and $n^{\prime}$ stand for the number of crossings in $D$ and $D^{\prime}$ respectively. Lemma 5.2 suggests which sign assignment to choose for $\mathcal{I}_{g r}\left(D \sqcup D^{\prime}\right)$, so that the corrected cube is isomorphic to $\mathcal{I}_{g r}^{\epsilon}(D) \wedge \nsucc \mathcal{I}_{g r}^{\epsilon^{\prime}}\left(D^{\prime}\right)$. Namely, we define $\epsilon \star \epsilon^{\prime} \in C^{1}\left(I^{n+n^{\prime}} ; \mathbb{k}^{*}\right)$ as follows:

$$
\begin{aligned}
& \left(\epsilon \star \epsilon^{\prime}\right)\left(\zeta \xi^{\prime}\right):=\epsilon(\zeta), \\
& \left(\epsilon \star \epsilon^{\prime}\right)\left(\xi \zeta^{\prime}\right):= \begin{cases}(-1)^{\|\xi\|} X^{a} Z^{b} \epsilon^{\prime}\left(\zeta^{\prime}\right), & \text { if } D_{\xi^{\prime}}^{\prime} \text { is a merge, } \\
(-1)^{\|\xi\|} Y^{b} Z^{-a} \epsilon^{\prime}\left(\zeta^{\prime}\right), & \text { if } D_{\xi^{\prime}}^{\prime} \text { is a split. }\end{cases}
\end{aligned}
$$

Notice that against the notation $\epsilon \star \epsilon^{\prime}$ depends on the diagram $D^{\prime}$. 
Lemma 5.3. The cochain $\epsilon \star \epsilon^{\prime}$ is a sign assignment for $\mathcal{I}_{g r}\left(D \sqcup D^{\prime}\right)$, for which the cube is isomorphic to $\mathcal{I}_{g r}^{\epsilon}(D) \downarrow \downarrow \mathcal{I}_{g r}^{\epsilon^{\prime}}\left(D^{\prime}\right)$.

Proof. Consider the following family of isomorphisms of vertices of the cubes:

$$
\begin{aligned}
&\left(\mathcal{I}_{g r}(D) \downarrow \downarrow \mathcal{I}_{g r}\left(D^{\prime}\right)\right)\left(\xi \xi^{\prime}\right)=D_{\xi}\{a, b\} \sqcup D_{\xi^{\prime}}\left\{a^{\prime}, b^{\prime}\right\} \\
& \cong i_{a+a^{\prime}, b+b^{\prime}}\left(i_{a, b}^{-1} \sqcup i_{a^{\prime}, b^{\prime}}^{-1}\right) \\
&\left(D_{\xi} \sqcup D_{\xi^{\prime}}^{\prime}\right)\left\{a+a^{\prime}, b+b^{\prime}\right\}=\mathcal{I}_{g r}\left(D \sqcup D^{\prime}\right)\left(\xi \xi^{\prime}\right)
\end{aligned}
$$

They form an isomorphism of cubes due to Lemma 5.2, so it remains to check $\epsilon \star \epsilon^{\prime}$ is indeed a sign assignment. This follows easily if $S$ is a face spanned by edges of $\mathcal{I}_{g r}(D)$ or $\mathcal{I}_{g r}\left(D^{\prime}\right)$, as $d\left(\epsilon \star \epsilon^{\prime}\right)(S)$ is equal then to $d \epsilon(S)$ or $d \epsilon\left(S^{\prime}\right)$ respectively. In a mixed case consider the diagram to the right, formed by the face $S$, its analogue in $\mathcal{I}_{g r}^{\epsilon}(D) \downarrow \downarrow \mathcal{I}_{g r}^{\epsilon^{\prime}}\left(D^{\prime}\right)$, and the isomorphisms (32). The four vertical squares commute due to Lemma 5.2, and the top horizontal square anticommutes. Hence, the face $S$ anticommutes (the bottom square), and as
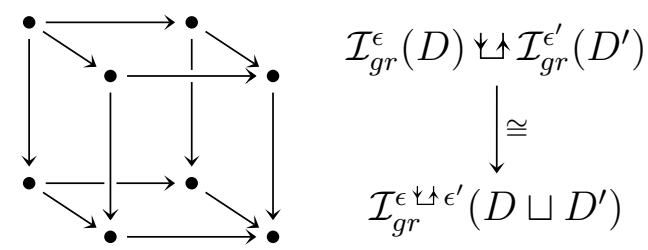
it represents a disjoint permutation relation (6) it must be $-d\left(\epsilon \star \epsilon^{\prime}\right)(S)=\psi(S)$.

Proposition 5.4. Given link diagrams $D$ and $D^{\prime}$ there is an isomorphism of bigraded complexes $K h\left(D \sqcup D^{\prime}\right)$ and $K h(D) \downarrow \Downarrow K h\left(D^{\prime}\right)$, which is natural with respect to graded morphisms.

Proof. The isomorphism of cubes from Lemma 5.3 implies that $\llbracket D \rrbracket_{\epsilon} \triangleq \Downarrow \llbracket D^{\prime} \rrbracket_{\epsilon^{\prime}} \cong \llbracket D \sqcup D^{\prime} \rrbracket_{\epsilon \nless \epsilon^{\prime}}$. The thesis follows, as a global degree shift does not affect the differential: $\lambda(a-b, x)=1$ in (26) if $a=b$.

There is a similar formula for another operation on links. The connected sum $D \# D^{\prime}$ of two oriented link diagrams with basepoints $D$ and $D^{\prime}$ is given by cutting the diagrams at the basepoints and gluing them in such a way that their orientations agree:

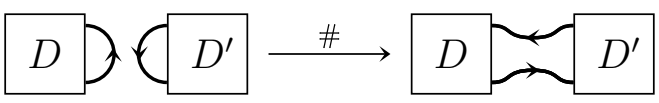

This operation does not depends on the exact placement of the basepoints, but only on the link components that carry them. Clearly, resolutions of $D \# D^{\prime}$ are exactly the connected sums of resolutions of $D$ and $D^{\prime}:\left(D \# D^{\prime}\right)_{\xi \xi^{\prime}}=D_{\xi} \# D_{\xi^{\prime}}$. Since the right connected sum \# behaves like the right disjoint union $k \downarrow$, in particular the Lemma 5.2 holds, the proofs of Lemma 5.3 and Proposition 5.4 can be easily adapted to this operation. We leave it to the reader to check the details.

Proposition 5.5. Given link diagram $D$ and $D^{\prime}$ there is an isomorphism of bigraded complexes $K h\left(D \# D^{\prime}\right) \cong K h(D) \# K h\left(D^{\prime}\right)$.

Consider now $(2 m, 2 n)$-tangles, i.e. embeddings of $n+m$ intervals and circles in $\mathbb{R}^{2} \times I$ with $2 n$ of the endpoints on $\mathbb{R}^{2} \times\{0\}$ and $2 m$ on $\mathbb{R}^{2} \times\{1\}$. Given a $(2 m, 2 n)$-tangle $T$ 
and a $(2 n, 2 k)$-tangle $T^{\prime}$ we can compose them by gluing along the $n$ edpoints to obtain a $(2 m, 2 k)$-tangle $T^{\prime} T$. In particular, a disjoint union of links correspond to composition of $(0,0)$ tangles, whereas a connected sum can be interpreted as a composition of a $(0,2)$ tangle with a $(2,0)$-tangle. From this viewpoint, Propositions 5.4 and 5.5 provide formulas for Khovanov complexes for compositions of $(2 m, 2 n)$-tangles with $m, n \leqslant 1$.

Unfortunately, the ideas from this paper do not immediately generalize to $m, n>1$. The main issue is the lack of distinction by the new degree of the two types of faces in a cube of resolutions labeled in Tab. 1 with 1 and $X Y$. Hence, for general tangles one needs more structure, which is a subject of future research.

\section{Module structure And homology}

The even Khovanov homology is known to carry a module structure over the Frobenius algebra associated to a circle [HN12, Kho04], and we want to obtain a similar result for the generalized complex. In order to have a universal result, independent of a chronological TQFT, we shall show $K h(D)$ is a module object over a certain algebra object on a circle.

Choose a symmetric monoidal category $\mathbf{C}$ with a unit $I$ and symmetry isomorphisms $c_{A, B}: A \otimes B \longrightarrow B \otimes A$.

Definition 6.1. An algebra object in $\mathbf{C}$ consists of an object $A$ and an associative morphism $m: A \otimes A \longrightarrow A$ called a product in $A$. We say that $(A, m)$ is symmetric if $m \circ c_{A, A}=m$, and unital if the diagram below commutes

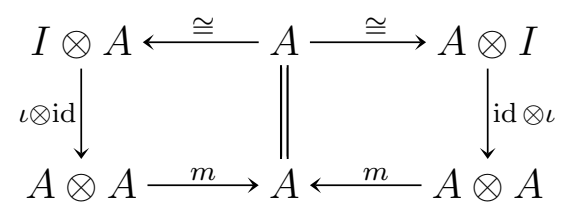

for some morphism $\iota: I \longrightarrow A^{3}{ }^{3}$

One example of an algebra object is provided by a circle $\bigcirc$ in the category $\mathbb{Z} \mathbf{C h C o b}$ with the merge cobordism as a product. It does not generalize immediately to $\mathbb{k} \mathbf{C h C o b}$, as the merge is not associative here. However, it is enough to shift the degree of $\bigcirc$ by $\{-1,0\}$ to obtain an associative product. It makes the merge a graded map.

Lemma 6.2. The merge cobordism induces on $\bigcirc\{-1,0\}$ a structure of a commutative unital algebra object.

Before we prove the lemma we shall extend our pictorial calculus of cobordisms by two degree shift isomorphisms: $9: A \longrightarrow A\{-1,0\}$ and $\theta$ : $A\{-1,0\} \longrightarrow A$. Both maps have nontrivial degrees, so that they commute with each other and with other generators only up to scaling by certain scalars controlled by the function $\lambda$. In particular, each time we switch heights of two bars we have to scale the cobordism by $X$.

\footnotetext{
${ }^{3}$ It can be shown that such a morphism is unique if it exists.
} 
Proof. Associativity follows from the chronological relations, and the fact that the two isomorphisms are inverse to each other:

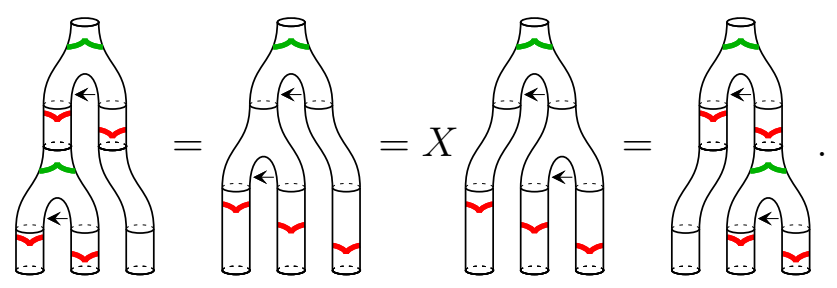

We left as an exercise to check the other axioms of an algebra object.

Definition 6.3. A left module object over a unital algebra object $A$ in $\mathbf{C}$ consists of an object $M$ and a morphism $a: A \otimes M \longrightarrow M$ such that the following diagram commutes:

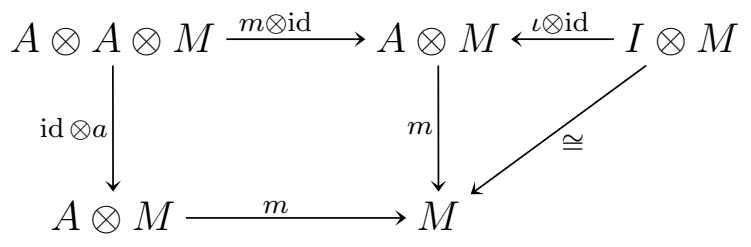

The morphism $a: A \otimes M \longrightarrow M$ is called an action of $A$ on $M$. Given two module objects $M$ and $N$ over $A$ a morphism $f: M \longrightarrow N$ in $\mathbf{C}$ is a homomorphism of module objects if it commutes with the actions, i.e. $m_{N} \circ(\mathrm{id} \otimes f)=f \circ m_{M}: A \otimes M \longrightarrow N$.

We define right module objects and homomorphisms between them likewise. Given two algebra objects $A$ and $B$ we say that $M$ is an $(A, B)$-bimodule object if it is a left $A$-module object, a right $B$-module object, and the two actions commute. An $(A, A)$-bimodule $M$ is symmetric if the diagram below commutes as well:

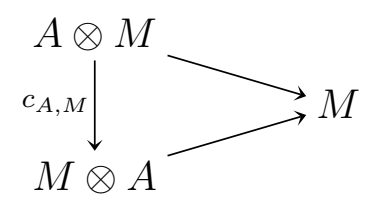

Any algebra object is at the same time a bimodule object over itself, and so is $\bigcirc\{-1,0\}$. We can generalize this to any family of circles, perhaps with a shifted degree, as long as we distinguish one of them. To show this we shall again extend our pictorial calculus by adding the pictures

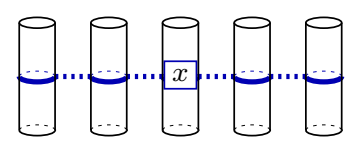

for the degree shift isomorphisms $(k \bigcirc)\{a\} \longrightarrow(k \bigcirc)\{a+x\}$, where $k \bigcirc$ stands for a disjoint union of $k$ circles and $a, x \in \mathbb{Z} \times \mathbb{Z}$. Clearly, we have the equalities

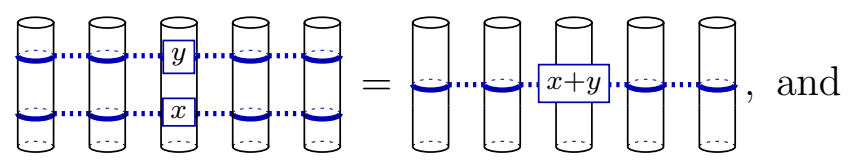




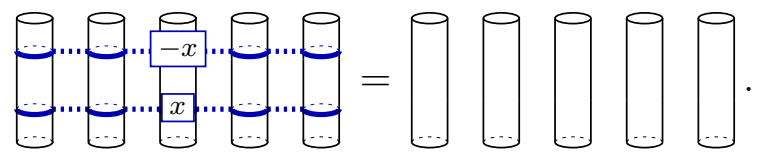

We distinguish one of the circles in $(k \bigcirc)\{a\}$ by placing a basepoint $p$ on it, and we define the left and right actions of $\bigcirc\{-1,0\}$ by merging the circle with $k \bigcirc$ at $p$ from the left and right hand side respectively, everything conjugated by the degree shift isomorphism accordingly:

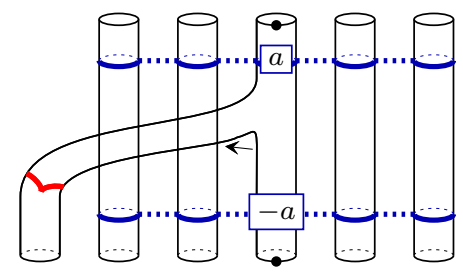

and

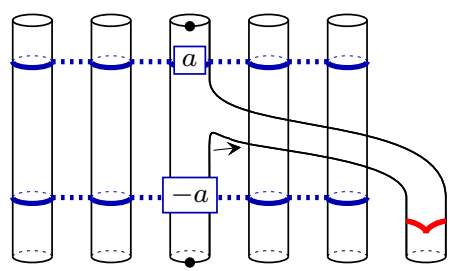

The pictures are not totally symmetric - compare the orders of degree shift isomorphisms.

Lemma 6.4. The above actions equip $k \bigcirc$ with a structure of a symmetric bimodule object over $\bigcirc\{-1,0\}$. In particular, given a based link diagram $L$ the cube $\mathcal{I}_{g r}(D)$ is a cube of symmetric bimodule objects and module homomorphisms.

Proof. All axioms of a symmetric bimodule for $k \bigcirc$ can be checked easily using the pictorial calculus of cobordism as in the proof of Lemma 6.2. Hence, vertices of $\mathcal{I}_{g r}(D)$ are decorated with symmetric bimodules objects. To show that an edge morphism preserves the left action of $\bigcirc\{-1,0\}$ we represent it in the following computation by a saddle conjugated with degree shift isomorphisms:

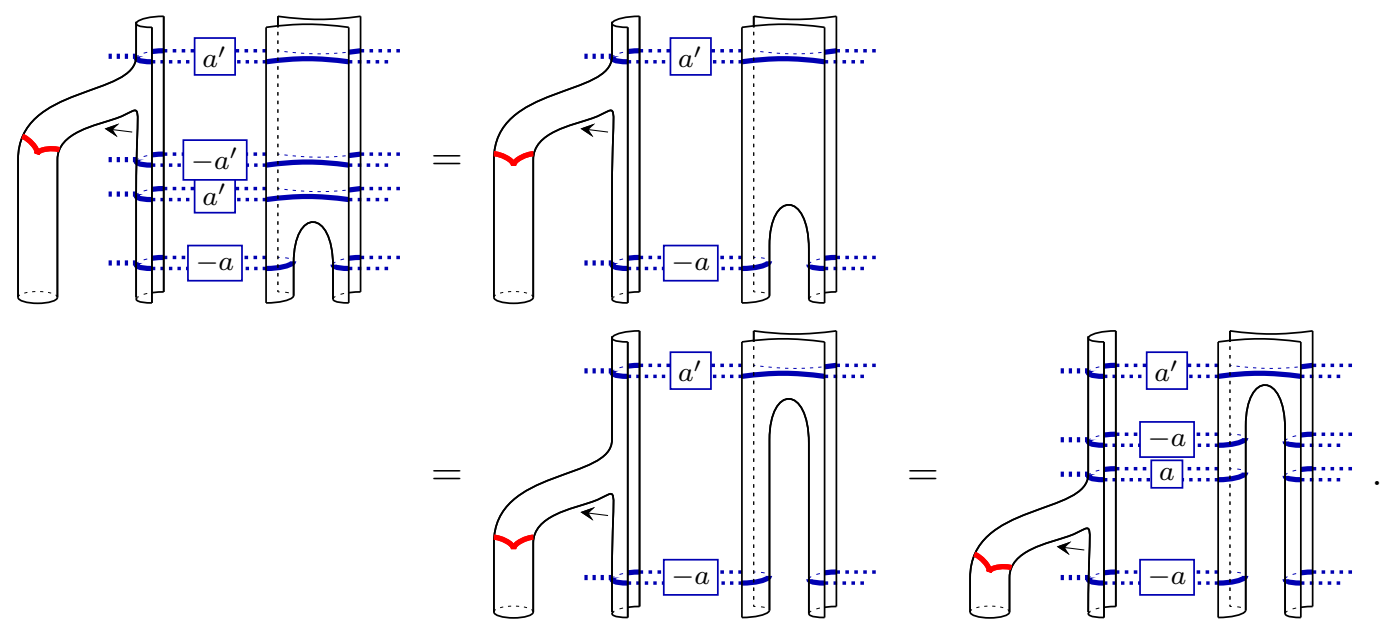

The case of the right action is proved likewise.

Corollary 6.5. The generalized Khovanov complex $K h(D)$ of a based link diagram $D$ is a complex of symmetric bimodule objects over $\bigcirc\{-1,0\}$.

The bimodule structure on $K h(D)$ is a link invariant. Indeed, one can always perform Reidemeister moves beyond a small neighborhood of the basepoint, perhaps using the isotopy through infinity, and the chain homotopy equivalences associated to these Reidemeister 
moves commute with the action of $\bigcirc\{-1,0\}$. In particular, we can move the basepoint freely along a component of a link.

On the other hand, moving a basepoint to a different component of a link may change the module structure. Following the idea from [HN12] we can choose a basepoint on every component of a link, which results in a bimodule structure on $K h(D)$ over the algebra object $\bigsqcup_{c}(\bigcirc\{-1,0\})$ consisting of as many copies of the shifted circle, as there are components in L. Again, this structure descends to homology, but we have to work harder to proof this structure is invariant under Reidemeister move: with more that one basepoint we cannot avoid passing them through crossings.

Theorem 6.6. Given a based link diagram D, the bimodule structure on $K h(D)$ is preserved up to isomorphism when the basepoint is moved through a crossing:

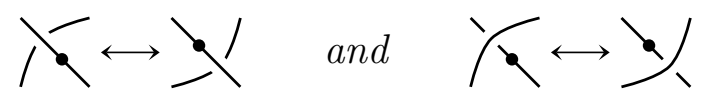

In particular, given a link $L$ with c components, the bimodule structure on $K h(L)$ over $\bigsqcup_{c}(\bigcirc\{-1,0\})$ is an invariant of $L$.

Proof. Invariance of the bimodule structure under passing a dot through a crossing is equivalent to saying that the following pairs of cobordisms

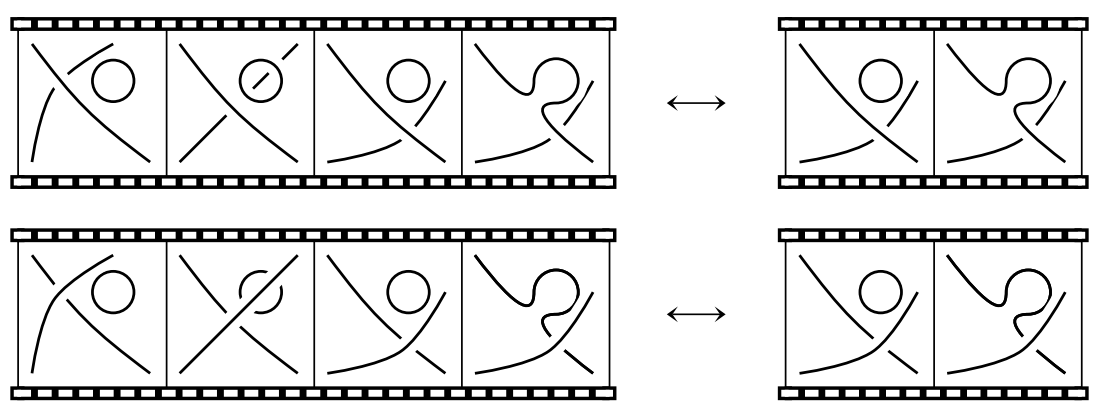

induce isomorphic operations on complexes. For that it is enough to show that placing a circle on one side of a strand is the same as placing it on the other side and moving over or under the piece of the link. We shall prove this only when the link diagram is the unknotthe general case then follows from Proposition 5.5, as every link $L$ with a basepoint is a connected sum of a based unknot with $L$.

We begin with computing the chain map induced by passing a circle over the unknot - it is given by a composition of the chain homotopy equivalences used in the proof of invariance of the chain complex under the second Reidemeister move - see the diagram in Fig. 3, in which we show only the essential fragment of the unknot. Instead of drawing the cobordisms, which would make the diagram illegible, we used the symbols $\mu, \Delta, \eta, \epsilon$ to denote respectively a merge, a split, a birth, and a death, with the exception of two morphisms that are of a particular interest to us. In addition we use the following conventions:

- the two crossings in (i) are decorated with arrows pointing inwards,

- when enumerating circles, the one being moved is always put first. 


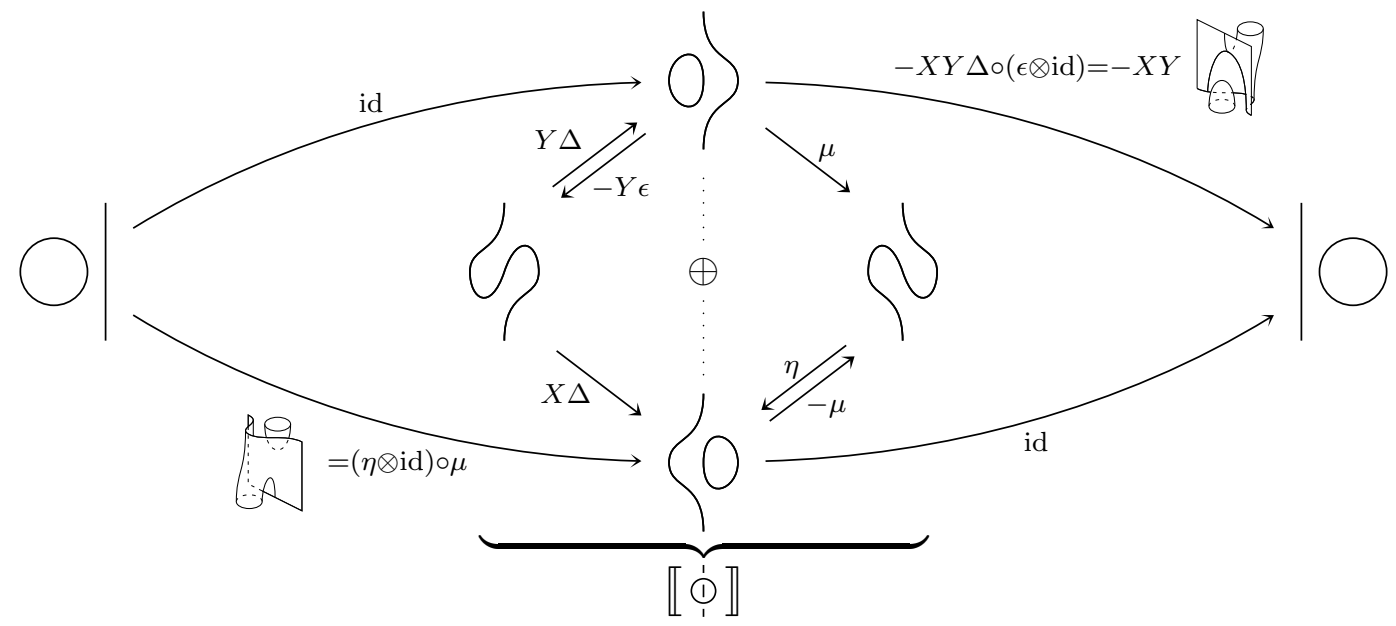

FiguRE 3. The chain map associated with a circle moving over a link diagram.

With the chosen decoration by arrows, the commutativity cocycle $\psi$ takes the value $X Y$ on the middle square, see Tab. 1 on page 9.

The left homomorphism is the inclusion $f: \llbracket \circ \mid \rrbracket \rightarrow \llbracket(1) \rrbracket$, and the right one is the retraction $g: \llbracket \uparrow \rrbracket \rightarrow \llbracket \mid \bigcirc \rrbracket$ from the proof of invariance of the complex under the second Reidemeister move; the two backward maps in the middle complex are pieces of chain homotopies (compare with Fig. 2). Regarding this diagram as in $\mathbb{k} \mathbf{C h C o b}$ and placing the circle acting on the knot, i.e. the one visible in full, as the left most one we compute the total map

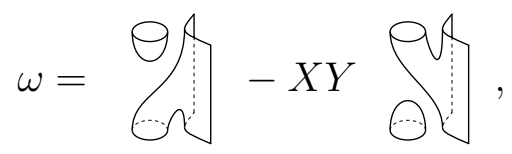

where again the convention is that the orienting arrows points towards left or back, and deaths are oriented clockwise. The expression (45) simplifies under the relation $4 \mathrm{Tu}$ into a disjoint union of a cobordism acting on a circle with a vertical wall:

$$
\begin{aligned}
& \Omega-X Y \text { O }=X Z^{-1}(x)
\end{aligned}
$$

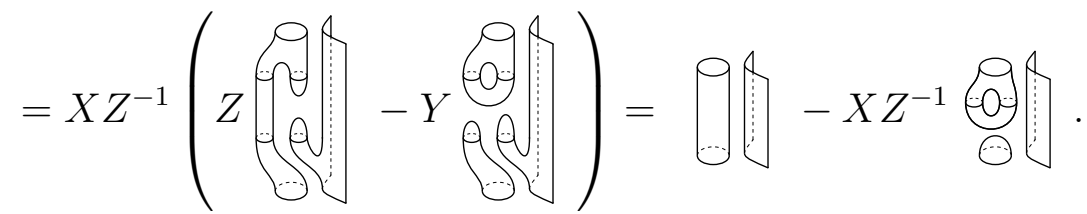

Denote by $\varphi: \bigcirc\{-1,0\} \longrightarrow \bigcirc\{-1,0\}$ the cobordism acting on the circle. A direct computation shows $\varphi^{2}=$ id

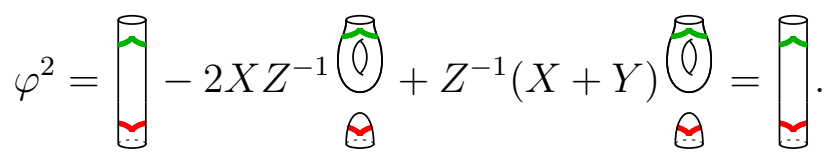


so that $\varphi$ is an isomorphism. In the above computation we used the fact that the polynomial $X-Y$ annihilates a punctured torus, see Theorem 2.4. It remains to check that it is a morphism of algebra objects, i.e. $\varphi \circ m=m \circ(\varphi \downarrow \Downarrow \varphi)$ where $m$ is the merge cobordism $m:=$ applied to the cobordism shown to the right. We left it to the reader to check

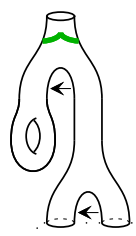
the details.

The other case, when a circle is moved below the unknot, results exactly in the same map.

Now choose your favorite chronological TQFT $\mathcal{F}$ that satisfies the relations $S, T$, and $4 T u$. It intertwines the disjoint union with the tensor product over $\mathbb{k}$, and the connected sum with a tensor product over the algebra $A^{\prime}:=\mathcal{F}(\bigcirc)\{1,0\}$; it follows directly from Lemma 6.2 that $A^{\prime}$ is an associative algebra.

A punctured torus vanishes in the odd Khovanov homology, making $\varphi=$ id. Hence, the action does not depend on the placement of the circle.

In the even case, one computes using the Khovanov's TQFT that $\mathcal{F} \varphi$ preserves $v_{+}$, but it takes $v_{-}$to $-v_{-}$. In other words, $\mathcal{F} \varphi$ is the conjugation on $A$ defined as $\bar{v}_{ \pm}:= \pm v_{ \pm}$. Hence, to define the action of the algebra $A:=\mathcal{F}(\bigcirc)$ properly, we can color the regions of the knot diagram $D$ black and white in a checkerboard manner; if the circle is merged to $D$ from a white region use the usual multiplication, but conjugate $A$ first if the circle is merged from a black region.

Lemma 6.4 together with Theorem 6.6 implies that given an $c$-component $\operatorname{link} L$ with a diagram $D$ the homology $\mathcal{H}(L):=H(\mathcal{F} K h(D))$ admits a module structure over the algebra $\left(A^{\prime}\right)^{\otimes c}$ that is independent on the diagram chosen. We are now ready to state the formulas for generalized Khovanov homology of composite links.

Theorem 6.7. Given two link diagrams $D, D^{\prime}$, and a chronological TQFT $\mathcal{F}$ there is an isomorphism of complexes $\mathcal{F} K h\left(D \sqcup D^{\prime}\right) \cong \mathcal{F} K h(D) \otimes \mathcal{F} K h\left(D^{\prime}\right)$. Moreover, if the diagrams are based, then $\mathcal{F} K h\left(D \# D^{\prime}\right) \cong \mathcal{F} K h(D) \underset{A^{\prime}}{\otimes} \mathcal{F} K h\left(D^{\prime}\right)$.

Proof. The first isomorphism follows from Proposition 5.4 and monoidality of $\mathcal{F}$. The second is a consequence of Proposition 5.5 and Corollary 6.5.

The sign assignment $\epsilon \star \epsilon^{\prime}$ we chose for $\mathcal{I}_{g r}\left(D \sqcup D^{\prime}\right)$ implies that

$$
d(x \otimes y)= \begin{cases}d x \otimes y+(-1)^{i} X^{a} Z^{b} x \otimes d y, & \text { if } d \otimes \text { id is a merge, } \\ d x \otimes y+(-1)^{i} Y^{a} Z^{b} x \otimes d y, & \text { if id } \otimes d \text { is a split, }\end{cases}
$$

for $x \otimes y \in \mathcal{F} \llbracket D \sqcup D^{\prime} \rrbracket \cong \mathcal{F} \llbracket D \rrbracket \otimes \mathcal{F} \llbracket D^{\prime} \rrbracket$. Since generalized Khovanov complexes are obtained from the brackets by not necessarily integral shifts, one must be careful with a choice of an isomorphism $\mathcal{F} \llbracket D \rrbracket \otimes \mathcal{F} \llbracket D^{\prime} \rrbracket \cong \mathcal{F} K h(D) \otimes \mathcal{F} K h\left(D^{\prime}\right)$. Indeed, taking a tensor product of 
the degree shift isomorphisms may require square roots of the generators $X, Y$, and $Z$. For example, in the following diagram

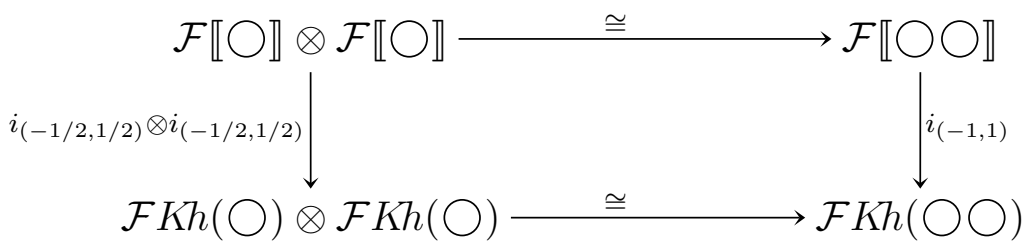

the bottom map takes $v_{+} \otimes v_{+}$into $X^{1 / 2} Z^{1 / 2} v_{+} \otimes v_{+}$. To overcome this problem, we replace the left isomorphism with the naive map $x \otimes y \longmapsto i(x) \otimes i^{\prime}(y)$ where $i: \llbracket D \rrbracket \longrightarrow K h(D)$ and $i: \llbracket D^{\prime} \rrbracket \longrightarrow K h\left(D^{\prime}\right)$ are the appropriate degree shift isomorphisms. Although not a canonical one, it is still a chain map.

Finally, the above discussion apply also to the connected sum of links. In particular, a similar formula to $(48)$ is true for the differential in $\mathcal{F} K h\left(D \# D^{\prime}\right)$.

\section{REFERENCES}

[Ab14] M. Abel, Virtual crossings and filtrations in Link Homology, Ph.D. Thesis, UNC-Chapel Hill, 2014. E-print: http://www. unc.edu/ maabel/Dissertation.pdf.

[BN02] D. Bar-Natan, On Khovanov's categorification of the Jones Polynomial, Alg. Geom. Topol. 2:337370, 2002. E-print: arXiv:math/0201043.

[BN05] D. Bar-Natan, Khovanov homology for tangles and cobordisms, Geom. Topol. 9:1443-1499, 2005. E-print: arXiv:math/0410495.

[HN12] M. Hedden, Y. Ni, Khovanov module and the detection of unlinks, 2012. E-print: arXiv:1204.0960.

[Kho99] M. Khovanov, A categorification of the Jones polynomial, Duke Math. J. 101(3):359-426, 2000. E-print: arXiv:math/9908171

[Kho04] M. Khovanov, Patters in Knot Cohomology, I, Experiment. Math. 12(3):365-374, 2003. E-print: arXiv:math/0201306

[ORS13] P. Ozsváth, J. Rasmussen, Z. Szabó, Odd Khovanov homology, 2007 Alg. Geom. Top. 13:1465-1488, 2013. E-print: arXiv:0710.4300.

[Put08] K. K. Putyra, Cobordisms with chronologies and a generalized Khovanov complex, Masters' Thesis, Jagiellonian University, 2008. E-print: arXiv:1004.0889.

[Put13] K. K. Putyra, A 2-category of chronological cobordisms and odd Khovanov homology, Banach Center Publications 103:287-351, 2015. Preprint: arXiv:1310.1895.

[Shu11] A. Shumakovitch, Patterns in odd Khovanov homology, J. Knot Theory Ramifications, 20:203-222, 2011. E-print: arXiv:1101.5607. 